\title{
Phenotyping for angular leaf spot severity and its implication in breeding common bean
}

\section{for resistance}

\author{
Rafael Pereira $^{1}$, Ângela de Fátima Barbosa Abreu ${ }^{2}$, Rafael Storto Nalin ${ }^{3}$, Elaine Aparecida de Souza ${ }^{*}$ (1)
}

${ }^{1}$ Universidade Federal de Lavras - Depto. de Biologia, C.P. 3037 - 37200-000 - Lavras, MG - Brasil.

2Embrapa Arroz e Feijão, Rod. GO-462, km 12 - 75375-000

- Santo Antônio de Goiás, GO - Brasil.

3Universidade de São Paulo/ESALQ - Depto. de Genética, Av. Pádua Dias, 11 - 13418-900 - Piracicaba, SP - Brasil. *Corresponding author <easouza@ufla.br>

Edited by: Roberto Fritsche Neto

Received December 02, 2017

Accepted April 26, 2018

\begin{abstract}
Phenotyping for severity of angular leaf spot (ALS) in common bean is important to identify new sources of resistance and select progenies in conventional and molecular markerassisted breeding. In this study, three phenotyping methods for ALS severity were assessed and its implications in breeding for resistance to Pseudocercospora griseola are discussed. Reaction of 144 common bean lines to $P$. griseola was evaluated. Three different experiments were conducted in the greenhouse (V2 and V3 stages) and in the field. Common bean lines were inoculated with a mixture of spores of pathogen races $63-63$ and $63-23$. We observed that $31 \%$, $7 \%$ and $10 \%$ of lines were resistant in stages V2, V3 and in the field, respectively. Estimates of coincidence index V2-V3, V2-field and V3-field were $68 \%, 69 \%$ and $88 \%$, respectively. Evaluations in V3 stage and in the field were the phenotyping methods more efficient for ALS severity. However, evaluation in V3 stage is impracticable to assess routinely many genotypes in breeding programs. Evaluation in V2 stage may be used to carry out a first screening, especially in the early stages of breeding programs. Moreover, this methodology may be used in association with evaluation of plants in the field to increase genetic gain.
\end{abstract}

Keywords: Phaseolus vulgaris L., Pseudocercospora griseola, genetic resistance, artificial inoculation

\section{Introduction}

Angular leaf spot (ALS) caused by the fungus Pseudocercospora griseola (Sacc) Crus \& U. Brown is one of the major diseases that affect common bean crops. Several studies have evaluated the variability of $P$. griseola and revealed predominance of race 63.63 isolates as well as the presence of variation within the same race (Rodriguez et al., 2014; Pereira et al., 2015). Studies on inheritance of common bean resistance to ALS have identified the presence of major genes and minor effect genes, however, quantitative (horizontal) resistance is predominant (Borel et al., 2011; Oblessuc et al., 2012; Pereira et al., 2015). Thus, recurrent selection is a suitable breeding method to obtain lines with durable resistance.

Recurrent selection is a strategy that has been used for this purpose at the Universidade Federal de Lavras (UFLA), in the state of Minas Gerais, Brazil. Evaluation of progenies, that is, ALS severity of plants, is carried out in the field in R7 stage, with natural occurrence of pathogen, in dry season. After 16 recurrent selection cycles, several lines presented resistance to $P$. griseola and therefore genomic regions responsible for qualitative and quantitative resistance have been established (Arantes, 2010; Rezende et al., 2014; Pereira et al., 2015).

Phenotyping ALS severity in plants is important to identify new resistance sources, select progenies in breeding programs, and identify quantitative trait loci (QTL) for a marker-assisted selection. Reliable phenotyping is a crucial step for accurate disease assessment (Oblessuc et al., 2012; Rezende et al., 2014). Plant phenotyping in the greenhouse has been performed by artificial inoculation of isolates of $P$. griseola (Silva et al., 2008; Pereira et al., 2013; Jara et al., 2015). Plants are inoculated in V3 stage according to the methodology proposed by Van Schoonhoven and Pastor-Corrales (1987).

Early evaluation of common bean plants, or V2 stage, has been proposed to evaluate simultaneously a large number of genotypes in germoplasm banks (Pereira et al., 2011; Librelon et al., 2015; Pádua et al., 2016). Many progenies are evaluated especially in early stages of breeding programs and plants evaluation in V3 stage is unfeasible. There are no reports that compare phenotyping in different stages/environments. In this study, three phenotyping methods for ALS severity were assessed and its implications in breeding for resistance to $P$. griseola are discussed.

\section{Materials and Methods}

Experiments were performed in the greenhouse and in the field, in the municipality of Lavras, state of Minas Gerais, Brazil. The altitude is $910 \mathrm{~m}$ and geographical coordinates are latitude $21^{\circ} 14^{\prime} \mathrm{S}$ and longitude $45^{\circ} 00^{\prime} \mathrm{W}$.

\section{Lines evaluated}

We evaluated 144 common bean lines from the Germplasm Bank of UFLA, including lines from value for cultivation and use testing (VCU) and lines from ten cycles of recurrent selection program for resistance to ALS. Lines BRSMG-Madrepérola, BRSMG-Majestoso, BRSMG-Talismã and Carioca MG were used as susceptible checks. 


\section{Isolates, mixture and preparations of suspensions}

This study used two strains of $P$. griseola belonging to races 63-63 and 63-23, collected at different locations in the state of Minas Gerais, Brazil, in 2011 (Pereira et al., 2015). Inoculum of each $P$. griseola strain was produced individually. Strains were grown on leaf-dextrose agar medium and kept at $24{ }^{\circ} \mathrm{C}$ in an incubator Biochemical Oxygen Demand (B.O.D) for 7 to $10 \mathrm{~d}$, with a photoperiod of $12 \mathrm{~h}$. Subsequently, the inoculum was prepared by adding $5-10 \mathrm{~mL}$ sterile distilled water to each culture dish and scraping the surface with a brush to release conidia. Conidial suspension was obtained by filtering through a layer of cheesecloth to remove mycelial fragments. Conidia were counted in a Neubauer chamber to standardize inoculum concentration to $2 \times$ $10^{4}$ conidia $\mathrm{mL}^{-1}$. Subsequently, conidial suspensions of both isolates were mixed at the ratio of $1: 1$.

\section{Phenotyping in the greenhouse}

\section{Phenotyping in V2 stage}

To evaluate ALS severity of lines, an experiment was conducted in a randomized block design (RBD) with three replications. Common bean lines were sown in polystyrene trays and the plot consisted of a row with nine plants. Eight days after sowing, seedlings were inoculated with a conidial suspension at a concentration of $2.0 \times 10^{4}$ conidia $\mathrm{mL}^{-1}$, using the methodology proposed by Pereira et al. (2011). Fifteen days after inoculation, disease severity was assessed using a 1 to 9-score scale proposed by Librelon et al. (2015): 1 - plants without disease symptoms; 2 - presence up to $2 \%$ of leaf lesions; 3 - presence up to $4 \%$ of leaf lesions; 4 - presence sporulating lesions covering $7 \%$ of leaf area; 5 - presence of several leaf lesions covering 7-16\% of leaf area; 6 - lesions covering between 16 and $26 \%$ of leaf area; 7 - lesions covering between $26-32 \%$ of leaf area; 8 - lesions covering $32-38 \%$ of leaf area; and 9 - severe symptoms of disease with lesions covering 26-32\% of leaf area.

\section{Phenotyping in V3 stage}

To evaluate ALS severity of lines, an experiment was conducted in RBD with three replications. The plot consisted of a pot containing $3 \mathrm{~kg}$ of soil in which four seeds were sown in each line. Conidial suspensions were inoculated after complete expansion of trifoliate leaves of plants by spraying both sides of leaves. After inoculation, plants were kept in the greenhouse, with relative humidity of $80 \%$, temperature around $24{ }^{\circ} \mathrm{C}$ for $15 \mathrm{~d}$ until evaluations. Disease severity was assessed using a 1 to 9-score scale of severity proposed by Van Schoonhoven and Pastor-Corrales (1987): 1 - plants without disease symptoms; 2 - presence up to $3 \%$ of leaf lesions; 3 - presence up to $5 \%$ of leaf lesions, without pathogen sporulation; 4 - presence of sporulating lesions covering $10 \%$ of leaf area; 5 - presence of various sporulating lesions between 2 and $3 \mathrm{~mm}$, covering $10-15 \%$ of leaf area; 6 - numerous sporulating lesions larger than 3 $\mathrm{mm}$, covering $15-20 \%$ of leaf area; 7 - numerous sporulating lesions larger than $3 \mathrm{~mm}$, covering between 20-25 $\%$ of leaf area; 8 - numerous sporulating lesions larger than $3 \mathrm{~mm}$, covering 25-30\% of leaf area; and 9 - severe disease symptoms, resulting in early leaf drop and plant death.

\section{Phenotyping in the field}

Common bean lines were sown on 4 Feb 2014 (dry season). The experimental design was $12 \times 12$ triple lattice and plots consisted of two rows of $1 \mathrm{~m}$. The crop was irrigated twice a week. Three artificial inoculations at 25, 30 and $40 \mathrm{~d}$ were carried out with a backpack sprayer. Pathogen inoculum was obtained as mentioned above. Severity of ALS in the field plots was evaluated 33 $\mathrm{d}$ after flowering (R7 stage) (Rezende et al., 2014) using the Van Schoonhoven and Pastor-Corrales (1987) scale.

\section{Data analysis}

Two different criteria were used to assess reaction to $P$. griseola of lines in the environments. In the first, lines were classified according to Van Schoonhoven and Pastor-Corrales (1987) and plants scoring 1 to 3 were considered resistant, and those scoring 3.1 or higher, susceptible. We estimated the coincidence index between the lines to compare the environments. Lines were considered coincident when there was no change in the classification of reaction (resistant or susceptible), regardless of the environment or evaluation stage.

The second criterion to evaluate lines for resistance was the joint analysis of score of ALS severity obtained in three assessment strategies using the mixed model approach. Components of variance estimates were obtained by Restricted Maximum Likelihood (REML) and prediction of genotypic values were obtained by Best Linear Unbiased Prediction (BLUP). The analyses were carried out in software $\mathrm{R}$ (R Core Team, version 3.2.3). For the individual analysis in each environment, we used genotypic univariate model, where: $y=B i+Z g+$ $e$, where, $y, i, g$ and $e$ are data vectors, effects of blocks and genotypes, and errors, respectively, (random), while $B$ and $Z$ are incidence matrices associated to vectors of effects $i$ and $g$, respectively. We assumed the effects of blocks genotypes and errors as random resulting in the following assumptions:

$$
\begin{aligned}
& i \mid \hat{\sigma}_{i}^{2} \sim N\left(0, I \hat{\sigma}_{i}^{2}\right) \\
& g \mid \hat{\sigma}_{g}^{2} \sim N\left(0, I \hat{\sigma}_{g}^{2}\right) \\
& e \mid \hat{\sigma}_{e}^{2} \sim N\left(0, I \hat{\sigma}_{e}^{2}\right)
\end{aligned}
$$

We used genotypic univariate model for the joint analysis, where: $y=B i+X b+Z g+W f+e$, where $y$, $i, b, g, f$ and $e$ are data vectors, effects of blocks, experiments, genotypes, interaction genotypes $\times$ experiments and errors; and $B, X, Z$ and $W$ are incidence matrices associated to vectors of effects $i, b, g$ and $f$, respectively. All effects were considered random, except for the effect of 
experiments. The following assumptions and covariance structures were used:

$$
\begin{aligned}
& i \mid \hat{\sigma}_{i}^{2} \sim N\left(0, I \hat{\sigma}_{i}^{2}\right) \\
& y \mid b, V \sim N(X b, V) \\
& g \mid \hat{\sigma}_{g}^{2} \sim N\left(0, I \hat{\sigma}_{g}^{2}\right) \\
& f \mid \hat{\sigma}_{g e}^{2} \sim N\left(0, I \hat{\sigma}_{g e}^{2}\right) \\
& e \mid \hat{\sigma}_{e}^{2} \sim N\left(0, D \hat{\sigma}_{e}^{2}\right)
\end{aligned}
$$

Error variances between experiments were considered heterogeneous and uncorrelated (diagonal matrix) in the joint analysis. Accuracy and genotypic variation coefficient were estimated according to Chiorato et al. (2008). Twenty per cent of lines with the lowest predicted genotypic values (BLUP averages) were considered as resistant. Genetic correlations between environments were estimated from predicted genotypic values in each experiment, using the Pearson correlation coefficients (Steel et al., 1997).

\section{Results}

\section{Genetic resistance of common bean lines to $P$. griseola in stages V2, V3 and in the field}

Number of resistant lines in stages V2, V3 and in the field were $44(31 \%), 10(7 \%)$ and $14(10 \%)$, respectively (Table 1).

Regarding the experiments in different environments and evaluation stages, coincidence percentage of lines, that is, lines that showed no change in phenotype (resistant or susceptible) was $63 \%$. Estimates of coincidence V2-V3, V2-field and V3-field were $68 \%, 69 \%$ and $88 \%$, respectively. Lines that presented resistance reaction in more than one stage or environment are listed in Table 2.

There was greater coincidence of lines with susceptibility reaction considering the reaction of lines in different stages/environments (Figure 1A and B).

Average scores for reaction of common bean lines to $P$. griseola showed higher frequency of scores 3.1 to 4 for V2 stage; however, for V3 stage and in the field, scores between 4.1 and 5 were more frequent (Figure 2).

\section{Genetic resistance of common bean lines to $P$. griseola on the average of stages/environments of evaluation.}

Estimates of variance components and genetic parameters for the three stages/environments (V2, V3 and in the field) and for the joint analysis are presented in Table 3. Estimates of genotypic coefficient of variation $\left(\mathrm{CV}_{\mathrm{g}}\right)$ and genetic variance components $\left(\hat{\sigma}_{g}^{2}\right)$ showed genetic variability among lines evaluated in all experiments. Thus, it is possible to infer the occurrence of different reaction levels of lines in all experiments and the joint analysis, when inoculated with mixture of strains of $P$. griseola.
Table 1 - Average scores for reaction of common bean lines to

\begin{tabular}{|c|c|c|c|c|c|c|c|}
\hline N. & Bean lines & $\mathrm{V} 2^{1}$ & V3 $3^{1}$ & Field ${ }^{1}$ & $\mathrm{~V} 2^{2}$ & $V^{2}{ }^{2}$ & Field $^{2}$ Joint $^{2}$ \\
\hline 1 & BRS Valente & $2.8 \mathrm{R}^{*}$ & $4.5 \mathrm{~S}$ & $5.7 \mathrm{~S}$ & $2.9 \mathrm{~S}$ & $4.5 \mathrm{~S}$ & $5.0 \mathrm{~S} 4.1 \mathrm{~S}$ \\
\hline 2 & BRS Timbó & $4.0 \mathrm{~S}$ & $5.4 \mathrm{~S}$ & $4.0 \mathrm{~s}$ & $3.9 \mathrm{~S}$ & $5.1 \mathrm{~S}$ & $4.6 \mathrm{~S} 4.5 \mathrm{~S}$ \\
\hline 3 & BRS Ametista & $4.3 \mathrm{~S}$ & $4.1 \mathrm{~S}$ & $4.0 \mathrm{~S}$ & $4.1 \mathrm{~S}$ & $4.2 \mathrm{~S}$ & $4.5 \mathrm{~S} 4.3 \mathrm{~S}$ \\
\hline 4 & BRS Campeiro & $4.2 \mathrm{~S}$ & $5.5 \mathrm{~S}$ & $5.3 \mathrm{~S}$ & $4.1 \mathrm{~S}$ & $5.3 \mathrm{~S}$ & $5.2 \mathrm{~S} 4.9 \mathrm{~S}$ \\
\hline 5 & BRS Esplendor & $2.5 \mathrm{R}$ & $4.9 \mathrm{~S}$ & $6.0 \mathrm{~s}$ & $2.8 \mathrm{R}$ & $4.7 \mathrm{~S}$ & $5.1 \mathrm{~S} 4.2 \mathrm{~S}$ \\
\hline 6 & BRS Esteio & $2.4 \mathrm{R}$ & $4.3 \mathrm{~S}$ & $6.0 \mathrm{~s}$ & $2.7 \mathrm{R}$ & $4.3 \mathrm{~S}$ & $5.0 \mathrm{~S} 4.0 \mathrm{~S}$ \\
\hline 7 & BRS Vereda & $4.0 \mathrm{~S}$ & $4.9 \mathrm{~S}$ & $3.7 \mathrm{~S}$ & $3.9 \mathrm{~S}$ & $4.7 \mathrm{~S}$ & $4.4 \mathrm{R} 4.3 \mathrm{~S}$ \\
\hline 8 & BRS Cometa & $4.9 \mathrm{~S}$ & $3.7 \mathrm{~S}$ & $4.0 \mathrm{~S}$ & $4.5 \mathrm{~S}$ & $4.0 \mathrm{~S}$ & $4.5 \mathrm{~S} \quad 4.4 \mathrm{~S}$ \\
\hline 9 & BRS Estilo & $4.0 \mathrm{~S}$ & $4.6 \mathrm{~S}$ & $4.7 \mathrm{~S}$ & $3.9 \mathrm{~S}$ & $4.6 \mathrm{~S}$ & $4.8 \mathrm{~S} \quad 4.4 \mathrm{~S}$ \\
\hline 10 & BRS Notável & $4.3 \mathrm{~S}$ & $5.3 \mathrm{~S}$ & $4.0 \mathrm{~S}$ & $4.1 \mathrm{~S}$ & $5.1 \mathrm{~S}$ & $4.6 \mathrm{~S} 4.6 \mathrm{~S}$ \\
\hline 11 & CNFP MG 11-18 & $3.4 \mathrm{~S}$ & $4.6 \mathrm{~S}$ & $5.3 \mathrm{~S}$ & $3.4 \mathrm{~S}$ & $4.5 \mathrm{~S}$ & $4.9 S 4.3 \mathrm{~S}$ \\
\hline 12 & CNFC 10108 & $4.7 \mathrm{~S}$ & $4.6 \mathrm{~S}$ & $5.5 \mathrm{~S}$ & $4.5 \mathrm{~S}$ & $4.7 \mathrm{~S}$ & $5.3 \mathrm{~S} 4.8 \mathrm{~S}$ \\
\hline 13 & CNFC & $4.4 \mathrm{~S}$ & $5.0 \mathrm{~S}$ & $3.7 \mathrm{~S}$ & $4.2 \mathrm{~S}$ & $4.9 \mathrm{~S}$ & $4.5 \mathrm{R} 4.5 \mathrm{~S}$ \\
\hline 14 & CNFC 10432 & $4.5 \mathrm{~S}$ & $4.9 \mathrm{~S}$ & $2.5 \mathrm{R}$ & $4.3 \mathrm{~S}$ & $4.7 \mathrm{~S}$ & $4.0 \mathrm{R} 4.3 \mathrm{~S}$ \\
\hline 15 & CNFC 11-07 & $4.4 \mathrm{~S}$ & $4.0 \mathrm{~S}$ & $5.0 \mathrm{~S}$ & $4.2 \mathrm{~S}$ & $4.2 \mathrm{~S}$ & $4.9 \mathrm{~S} 4.4 \mathrm{~S}$ \\
\hline 16 & CNFC 11946 & $5.0 \mathrm{~S}$ & $4.4 \mathrm{~S}$ & $6.3 \mathrm{~S}$ & $4.7 \mathrm{~S}$ & $4.6 \mathrm{~S}$ & $5.7 \mathrm{~S} 5.3 \mathrm{~S}$ \\
\hline 17 & CNFC & $3.6 \mathrm{~S}$ & $5.2 \mathrm{~S}$ & $5.3 \mathrm{~S}$ & $3.6 \mathrm{~S}$ & $4.9 \mathrm{~S}$ & $5.0 \mathrm{~S} 4.5 \mathrm{~S}$ \\
\hline 18 & CNFCMG 11-08 & $3.0 \mathrm{R}$ & $4.2 \mathrm{~S}$ & $3.3 \mathrm{~S}$ & $3.0 \mathrm{~s}$ & $4.2 \mathrm{~S}$ & $4.0 \mathrm{R} \quad 3.7 \mathrm{~S}$ \\
\hline 19 & CNFCMG 11-13 & $4.0 \mathrm{~S}$ & $4.2 \mathrm{~S}$ & $5.7 \mathrm{~S}$ & $3.9 \mathrm{~S}$ & $4.3 \mathrm{~S}$ & $5.1 \mathrm{~S} 4.4 \mathrm{~S}$ \\
\hline 20 & CNFJ 15288 & $3.7 \mathrm{~S}$ & $4.5 \mathrm{~S}$ & $4.3 \mathrm{~S}$ & $3.6 \mathrm{~S}$ & $4.5 \mathrm{~S}$ & $4.6 \mathrm{~S} 4.2 \mathrm{~S}$ \\
\hline 21 & CNFP N & $3.6 \mathrm{~S}$ & $4.3 \mathrm{~S}$ & $5.0 \mathrm{~S}$ & $3.5 \mathrm{~S}$ & $4.4 \mathrm{~S}$ & $4.8 \mathrm{~S} \quad 4.4 \mathrm{~S}$ \\
\hline 22 & CNFP & $3.2 \mathrm{~S}$ & $4.5 \mathrm{~S}$ & $4.7 \mathrm{~S}$ & $3.3 \mathrm{~S}$ & $4.4 \mathrm{~S}$ & $4.6 \mathrm{~S} 4.9 \mathrm{~S}$ \\
\hline 23 & CNFPMG -1106 & $2.6 \mathrm{R}$ & $4.8 \mathrm{~S}$ & $5.7 \mathrm{~S}$ & $2.8 \mathrm{R}$ & $4.6 \mathrm{~S}$ & $5.0 \mathrm{~S} 4.1 \mathrm{~S}$ \\
\hline 24 & CNFPMG -11-21 & $3.0 \mathrm{R}$ & $4.7 \mathrm{~S}$ & $4.7 \mathrm{~S}$ & $3.1 \mathrm{~S}$ & $4.5 \mathrm{~S}$ & $4.6 \mathrm{~S} 4.1 \mathrm{~S}$ \\
\hline 25 & CNFRX 15275 & $4.2 \mathrm{~S}$ & $4.5 \mathrm{~S}$ & $5.7 \mathrm{~S}$ & $4.0 \mathrm{~S}$ & $4.5 \mathrm{~S}$ & $5.2 \mathrm{~S} 4.6 \mathrm{~S}$ \\
\hline 26 & CVIII 6 & $3.8 \mathrm{~S}$ & $4.3 \mathrm{~S}$ & $5.0 \mathrm{~S}$ & $3.7 \mathrm{~S}$ & $4.4 \mathrm{~S}$ & $4.8 \mathrm{~S} 4.3 \mathrm{~S}$ \\
\hline 27 & CVIII 2 & $2.0 \mathrm{R}$ & $4.7 \mathrm{~S}$ & $4.5 \mathrm{~S}$ & $2.3 \mathrm{R}$ & $4.4 \mathrm{~S}$ & $4.4 \mathrm{~S} 3.7 \mathrm{~S}$ \\
\hline 28 & CVIII 5 & $4.3 \mathrm{~S}$ & $5.3 \mathrm{~S}$ & $3.5 \mathrm{~S}$ & $4.1 \mathrm{~S}$ & $5.1 \mathrm{~S}$ & $4.4 \mathrm{R} 4.6 \mathrm{~S}$ \\
\hline 29 & $C X$ & $2.7 \mathrm{R}$ & $3.3 \mathrm{~S}$ & $4.3 \mathrm{~S}$ & $2.7 \mathrm{R}$ & $3.5 \mathrm{R}$ & $4.2 \mathrm{~S} \quad 3.5 \mathrm{R}$ \\
\hline 30 & CXI 18 & $4.3 \mathrm{~S}$ & $4.7 \mathrm{~S}$ & $3.0 \mathrm{R}$ & $4.1 \mathrm{~S}$ & $4.6 \mathrm{~S}$ & $4.2 \mathrm{R} 4.3 \mathrm{~S}$ \\
\hline 31 & CXI 7 & $3.8 \mathrm{~S}$ & $3.7 \mathrm{~S}$ & $5.7 \mathrm{~S}$ & $3.7 \mathrm{~S}$ & $4.0 \mathrm{~S}$ & $5.0 \mathrm{~S} 4.5 \mathrm{~S}$ \\
\hline 32 & Carioca & $4.4 \mathrm{~S}$ & $4.6 \mathrm{~S}$ & $5.3 \mathrm{~S}$ & $4.3 \mathrm{~S}$ & $4.7 \mathrm{~S}$ & $5.1 \mathrm{~S} 4.7 \mathrm{~S}$ \\
\hline 33 & Carioca MG & $4.4 \mathrm{~S}$ & $4.7 \mathrm{~S}$ & $5.0 \mathrm{~S}$ & $3.1 \mathrm{~S}$ & $4.5 \mathrm{~S}$ & $4.7 \mathrm{~S} 4.1 \mathrm{~S}$ \\
\hline 34 & CNFCMG-11-06 & $3.8 \mathrm{~S}$ & $4.1 \mathrm{~S}$ & $4.3 \mathrm{~S}$ & $3.7 \mathrm{~S}$ & $4.2 \mathrm{~S}$ & $4.5 \mathrm{~S} 4.1 \mathrm{~S}$ \\
\hline 35 & CXI-26 & $3.7 \mathrm{~S}$ & $4.4 \mathrm{~S}$ & $5.7 \mathrm{~S}$ & $3.7 \mathrm{~S}$ & $4.5 \mathrm{~S}$ & $5.1 \mathrm{~S} 4.4 \mathrm{~S}$ \\
\hline 36 & CXII 19 & $4.4 \mathrm{~S}$ & $4.2 \mathrm{~S}$ & $5.7 \mathrm{~S}$ & $4.2 \mathrm{~S}$ & $4.4 \mathrm{~S}$ & $5.2 \mathrm{~S} 4.6 \mathrm{~S}$ \\
\hline 37 & CXII-1 & $4.4 \mathrm{~S}$ & $4.7 \mathrm{~S}$ & $6.0 \mathrm{~s}$ & $4.3 \mathrm{~S}$ & $4.8 \mathrm{~S}$ & $5.4 \mathrm{~S} 4.8 \mathrm{~S}$ \\
\hline 38 & CXII-13 & $4.0 \mathrm{~S}$ & $4.2 \mathrm{~S}$ & $6.0 \mathrm{~s}$ & $4.0 \mathrm{~S}$ & $4.3 \mathrm{~S}$ & $5.3 \mathrm{~S} 4.5 \mathrm{~S}$ \\
\hline 39 & CXII-15 & $2.8 \mathrm{R}$ & $2.9 R$ & $6.0 \mathrm{~s}$ & $2.9 \mathrm{~S}$ & $3.4 \mathrm{R}$ & $4.9 \mathrm{~S} 3.7 \mathrm{~S}$ \\
\hline 40 & CXII-23 & $4.1 \mathrm{~S}$ & $4.4 \mathrm{~S}$ & $6.3 \mathrm{~S}$ & $4.0 \mathrm{~S}$ & $4.5 \mathrm{~S}$ & $5.4 \mathrm{~S} 4.7 \mathrm{~S}$ \\
\hline 41 & CXII-6 & $4.1 \mathrm{~S}$ & $4.6 \mathrm{~S}$ & $5.0 \mathrm{~S}$ & $4.0 \mathrm{~S}$ & $4.6 \mathrm{~S}$ & $4.9 \mathrm{~S} 4.5 \mathrm{~S}$ \\
\hline 42 & CXII-8 & $3.9 \mathrm{~S}$ & $4.2 \mathrm{~S}$ & $6.7 \mathrm{~S}$ & $3.9 \mathrm{~S}$ & $4.4 \mathrm{~S}$ & $5.5 \mathrm{~S} \quad 4.6 \mathrm{~S}$ \\
\hline 43 & CXII 16 & $3.4 \mathrm{~S}$ & $3.6 \mathrm{~S}$ & $3.7 \mathrm{~S}$ & $3.3 \mathrm{~S}$ & $3.8 \mathrm{R}$ & $4.1 \mathrm{R} 3.7 \mathrm{~S}$ \\
\hline 44 & E 09/10-8 & $3.9 \mathrm{~S}$ & $4.3 \mathrm{~S}$ & $4.7 \mathrm{~S}$ & $3.8 \mathrm{~S}$ & $4.4 \mathrm{~S}$ & $4.7 \mathrm{~S} 4.3 \mathrm{~S}$ \\
\hline 45 & E09/ 10-27 & $3.9 \mathrm{~S}$ & $4.7 \mathrm{~S}$ & $5.0 \mathrm{~S}$ & $3.8 \mathrm{~S}$ & $4.6 \mathrm{~S}$ & $4.9 \mathrm{~S} \quad 4.4 \mathrm{~S}$ \\
\hline 46 & E09/10-5 & $4.2 \mathrm{~S}$ & $4.3 \mathrm{~S}$ & $5.3 \mathrm{~S}$ & $4.1 \mathrm{~S}$ & $4.4 \mathrm{~S}$ & $5.1 \mathrm{~S} 4.5 \mathrm{~S}$ \\
\hline 47 & E09/10-28 & $3.3 \mathrm{~S}$ & $4.1 \mathrm{~S}$ & $5.3 \mathrm{~S}$ & $3.3 \mathrm{~S}$ & $4.2 \mathrm{~S}$ & $4.9 \mathrm{~S} \quad 4.4 \mathrm{~S}$ \\
\hline 48 & E09/10-7 & $3.9 \mathrm{~S}$ & $4.4 \mathrm{~S}$ & $6.0 \mathrm{~S}$ & $3.9 \mathrm{~S}$ & $4.5 \mathrm{~S}$ & $5.3 \mathrm{~S} 4.6 \mathrm{~S}$ \\
\hline 49 & EMB9 & $4.1 \mathrm{~S}$ & $4.7 \mathrm{~S}$ & $4.0 \mathrm{~S}$ & $3.9 \mathrm{~S}$ & $4.6 \mathrm{~S}$ & $4.5 \mathrm{~S} 4.3 \mathrm{~S}$ \\
\hline 50 & IPR Uirapuru & $3.4 \mathrm{~S}$ & $5.0 \mathrm{~S}$ & $3.0 \mathrm{R}$ & $3.4 \mathrm{~S}$ & $4.7 \mathrm{~S}$ & $4.2 \mathrm{R} 4.1 \mathrm{~S}$ \\
\hline 51 & Madrepérola & $4.2 \mathrm{~S}$ & $4.4 \mathrm{~S}$ & $4.7 \mathrm{~S}$ & $4.1 \mathrm{~S}$ & $4.5 \mathrm{~S}$ & $4.8 \mathrm{~S} 4.4 \mathrm{~S}$ \\
\hline 52 & MAl- $2.5^{*}$ & $4.7 \mathrm{~S}$ & $7.0 \mathrm{~S}$ & $4.5 \mathrm{~S}$ & $4.6 \mathrm{~S}$ & $6.3 \mathrm{~S}$ & $5.1 \mathrm{~S} 5.3 \mathrm{~S}$ \\
\hline 53 & MAI-18.13** & $2.7 \mathrm{R}$ & $4.7 \mathrm{~S}$ & $2.0 \mathrm{R}$ & $2.8 \mathrm{~S}$ & $4.4 \mathrm{~S}$ & $3.5 \mathrm{R} \quad 3.6 \mathrm{R}$ \\
\hline
\end{tabular}
Pseudocercospora griseola in different stages/environments of evaluation. Predicted genotypic values (average Best Linear Unbiased Prediction) and reaction of common bean lines to $P$. griseola, assessed in different environments/stages.

Continue 
Table 1 - Continuation.

\begin{tabular}{|c|c|c|c|c|c|c|c|}
\hline & & & & & & & \\
\hline & Al-6.10" & $\mathrm{S}$ & $.9 \mathrm{~S}$ & $4.0 \mathrm{~S}$ & 5 & $4.7 \mathrm{~S}$ & \\
\hline & & & $5 \mathrm{~S}$ & $0 \mathrm{~S}$ & $1 \mathrm{~s}$ & $.4 \mathrm{~S}$ & \\
\hline & & & $S$ & $3 S$ & $2 \mathrm{~S}$ & $4.7 \mathrm{~S}$ & $R$ \\
\hline & $11-10^{* *}$ & $0 \mathrm{R}$ & $.0 \mathrm{~S}$ & $2.7 \mathrm{R}$ & $3.0 \mathrm{~S}$ & $4.0 \mathrm{~S}$ & $.7 R$ \\
\hline & MAll-16 & $1 \mathrm{~S}$ & $4 \mathrm{~S}$ & $.0 \mathrm{~S}$ & $3.2 \mathrm{~S}$ & $4.4 \mathrm{~S}$ & $.7 \mathrm{~s}$ \\
\hline & & $7 \mathrm{~S}$ & $.0 \mathrm{R}$ & $3.7 \mathrm{~S}$ & $3.6 \mathrm{~S}$ & $3.4 \mathrm{R}$ & 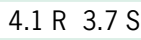 \\
\hline & NIII-16.15 & R & $3 S$ & $5 \mathrm{R}$ & $3.0 \mathrm{~S}$ & $3.5 \mathrm{R}$ & $5 \mathrm{R}$ \\
\hline & 116 & $S$ & $8 \mathrm{~S}$ & $.0 \mathrm{~S}$ & $6 \mathrm{~S}$ & $4.1 \mathrm{~S}$ & $6 \mathrm{~s}$ \\
\hline & MAll-17.15 & $2 S$ & $3 \mathrm{~S}$ & $.0 \mathrm{~S}$ & $4.0 \mathrm{~S}$ & $4.3 \mathrm{~S}$ & $.5 \varsigma$ \\
\hline & & $2 S$ & $3.6 \mathrm{~S}$ & $2.7 \mathrm{R}$ & & $3.7 \mathrm{R}$ & .7 \\
\hline & 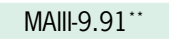 & & & $5 \mathrm{R}$ & & $3.6 \mathrm{R}$ & 政 \\
\hline & $v_{1}$ & $\mathrm{R}$ & $7 S$ & $7 \mathrm{~S}$ & $3 \mathrm{R}$ & $3.7 \mathrm{R}$ & 3 \\
\hline & 1 & $8 \mathrm{~S}$ & $7 \mathrm{R}$ & $.7 \mathrm{R}$ & $3.6 \mathrm{~S}$ & $3.2 \mathrm{R}$ & 6 \\
\hline & & $5 \mathrm{R}$ & $3 R$ & $.0 \mathrm{~S}$ & $2.5 \mathrm{R}$ & $2.8 \mathrm{R}$ & .9 \\
\hline & V-10.2. & S & 45 & & $2.9 \mathrm{~S}$ & $3.0 \mathrm{~K}$ & 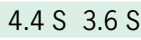 \\
\hline & 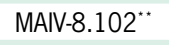 & $S$ & $7 \mathrm{R}$ & $3 R$ & $3.1 \mathrm{~S}$ & $3.1 \mathrm{R}$ & $.4 \mathrm{~F}$ \\
\hline & & $9 \mathrm{R}$ & $4 \mathrm{~S}$ & $7 \mathrm{~S}$ & $3.0 \mathrm{~S}$ & $4.3 \mathrm{~S}$ & $6 \mathrm{~s}$ \\
\hline & & $6 \mathrm{~S}$ & $9 \mathrm{~S}$ & $0 \mathrm{~S}$ & $3.6 \mathrm{~S}$ & $4.0 \mathrm{~S}$ & 4.7 \\
\hline & M & & $9 S$ & $.0 \mathrm{~S}$ & $3.2 \mathrm{~S}$ & $4.0 \mathrm{~S}$ & .7 \\
\hline & & & & & $R$ & & 25 \\
\hline & & & & & & $3.9 \mathrm{~S}$ & 253 \\
\hline & Mai & $S$ & & $3 S$ & $3.7 \mathrm{~S}$ & $5.1 \mathrm{~S}$ & 4.7 \\
\hline & & & & & $3.7 \mathrm{~S}$ & S & $\Gamma$ \\
\hline & & & & & $3.5 \mathrm{~S}$ & 35 & .95 \\
\hline & & & S & $7 \mathrm{~S}$ & $3.3 \mathrm{~s}$ & $3 \mathrm{~S}$ & $2 \mathrm{R}$ \\
\hline & & $5 \mathrm{R}$ & $3 S$ & $.0 \mathrm{R}$ & $2.7 \mathrm{R}$ & $4.8 \mathrm{~S}$ & $3.9 \mathrm{R} 3$ \\
\hline & & $R$ & $1 \mathrm{~S}$ & $3 S$ & $2.4 \mathrm{R}$ & $3.3 \mathrm{R}$ & $3.7 \mathrm{~F}$ \\
\hline & & & 35 & S & IS & $5.0 \mathrm{~S}$ & $.5 \mathrm{R}$ \\
\hline & & & & & & $S$ & $0 \mathrm{~S}$ \\
\hline & & $\mathrm{R}$ & $S$ & $S$ & $8 R$ & $3.9 \mathrm{~S}$ & 2 \\
\hline & & & & $S$ & $3.0 \mathrm{~S}$ & $3.9 \mathrm{~S}$ & $4.6 \mathrm{~S}$ \\
\hline & & & & & & & $4.1 \mathrm{r}$ \\
\hline & & & & & $2.5 \mathrm{R}$ & & $9 \mathrm{R}$ \\
\hline & & $\mathrm{R}$ & $5 \mathrm{~S}$ & $3 S$ & $2.2 \mathrm{R}$ & $R$ & $.7 \mathrm{R} 3$ \\
\hline & & & & $S$ & $5 S$ & $S$ & $4 \mathrm{~S}$ \\
\hline & & & & $R$ & $2.6 \mathrm{R}$ & & $1 \mathrm{R} 3$ \\
\hline & & & & O S & $2.4 \mathrm{R}$ & $\mathrm{R}$ & 45 \\
\hline & & & & R & $R$ & $S$ & $.7 \mathrm{R}$ \\
\hline & & & & S & $3 R$ & $S$ & .55 \\
\hline & & & & $S$ & $2.6 \mathrm{R}$ & & $4.6 \mathrm{~S} 3$ \\
\hline & & & & & $4 \mathrm{R}$ & & 5 \\
\hline & & & & & $2 R$ & & \\
\hline & & & & S & $2.7 \mathrm{R}$ & $7 \mathrm{R}$ & $4 \mathrm{~S}$ \\
\hline & & & $S$ & $7 S$ & $2.5 \mathrm{R}$ & $\mathrm{S}$ & $.8 \mathrm{~s} 3$. \\
\hline & & & & $3 S$ & $3.2 \mathrm{~S}$ & & $4 \mathrm{~S} 3$ \\
\hline & & & $S$ & $7 \mathrm{~S}$ & $3.9 \mathrm{~S}$ & $R$ & $2 \mathrm{R} 4$. \\
\hline & & & & & $.7 \mathrm{R}$ & & 45 \\
\hline & & & & & $6 \mathrm{R}$ & & $5 \mathrm{~S} 3$. \\
\hline & & & & $S$ & $2 S$ & & $4 \mathrm{~S} 3$ \\
\hline & & & & & $2.4 \mathrm{R}$ & & \\
\hline & & & & & & & \\
\hline & & & & $7 \mathrm{~S}$ & $9 \mathrm{~S}$ & $3.7 \mathrm{R}$ & 1.0 \\
\hline & & & & $\mathrm{S}$ & $3.4 \mathrm{~S}$ & $S$ & 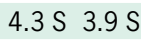 \\
\hline & & & & $1 \mathrm{~S}$ & $7 \mathrm{~S}$ & & \\
\hline & & & & 3 & 35 & & \\
\hline & & & $7 \mathrm{~S}$ & & & $4.0 \mathrm{R}$ & \\
\hline & MBCO-22/3 & $R$ & $2 S$ & $0 \mathrm{~S}$ & $3.0 \mathrm{~S}$ & $4.2 \mathrm{~S}$ & \\
\hline & uro Vermelho & $2.7 \mathrm{R}$ & $5.4 \mathrm{~S}$ & $6.3 \mathrm{~S}$ & $2.9 \mathrm{~S}$ & $5.1 \mathrm{~S}$ & 3 \\
\hline
\end{tabular}

113 Ouro Negro $3.9 \mathrm{~S} \quad 4.6 \mathrm{~S} \quad 5.7 \mathrm{~S} \quad 3.8 \mathrm{~S} \quad 4.6 \mathrm{~S} \quad 5.1 \mathrm{~S} \quad 4.5 \mathrm{~S}$

114 BRSMG Pérola $4.1 \mathrm{~S} \quad 4.6 \mathrm{~S} \quad 6.0 \mathrm{~S} \quad 4.0 \mathrm{~S} \quad 4.6 \mathrm{~S} \quad 5.3 \mathrm{~S} \quad 4.7 \mathrm{~S}$ $\begin{array}{lllllllll}115 & \mathrm{PT} 65 & 2.1 \mathrm{R} & 2.7 \mathrm{R} & 2.7 \mathrm{R} & 2.2 \mathrm{R} & 3.0 \mathrm{R} & 3.4 \mathrm{R} & 2.9 \mathrm{R}\end{array}$

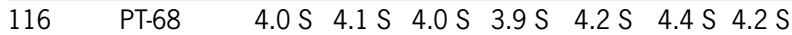

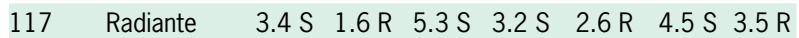

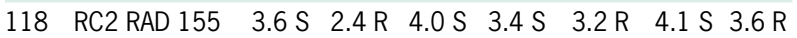

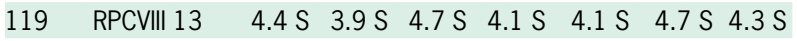

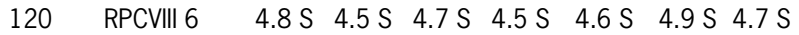
$\begin{array}{lllllllll}121 & \text { RPCVIII } 7 & 3.7 \mathrm{~S} & 4.6 \mathrm{~S} & 4.3 \mathrm{~S} & 3.7 \mathrm{~S} & 4.5 \mathrm{~S} & 4.6 \mathrm{~S} & 4.2 \mathrm{~S}\end{array}$

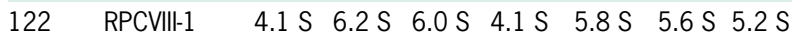
$123 \quad$ RPCVIII-4 $4.1 \mathrm{~S} \quad 5.5 \mathrm{~S} \quad 6.3 \mathrm{~S} \quad 4.1 \mathrm{~S} \quad 5.2 \mathrm{~S} \quad 5.6 \mathrm{~S} \quad 5.0 \mathrm{~S}$ $124 \quad$ RPCVIII-8 $3.6 \mathrm{~S} \quad 4.3 \mathrm{~S} \quad 6.0 \mathrm{~S} \quad 3.6 \mathrm{~S} \quad 4.4 \mathrm{~S} \quad 5.2 \mathrm{~S} \quad 4.4 \mathrm{~S}$ 125 BRSMG Talismã $3.9 \mathrm{~S} \quad 4.4 \mathrm{~S} \quad 5.0 \mathrm{~S} \quad 3.8 \mathrm{~S} \quad 4.4 \mathrm{~S} \quad 4.9 \mathrm{~S} \quad 4.4 \mathrm{~S}$

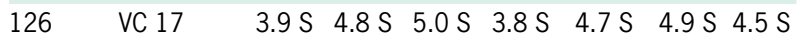

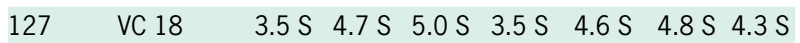
$128 \quad V C 19 \quad 4.0 S \quad 4.6 S \quad 4.0 S \quad 3.8 S \quad 4.5 S \quad 4.5 S \quad 4.3 S$ $129 \quad V C 21 \quad 3.2 S \quad 5.5 S \quad 6.3 S \quad 3.3 S \quad 5.2 S \quad 5.4 S \quad 4.6 S$

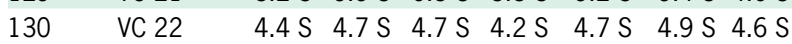

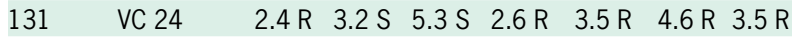

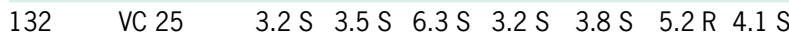

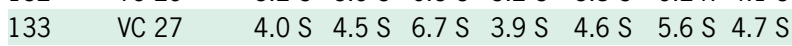

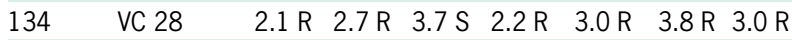
$\begin{array}{lllllllll}135 & V C 20 & 2.1 R & 3.8 S & 3.3 \mathrm{~S} & 2.3 \mathrm{R} & 3.8 \mathrm{~S} & 3.8 \mathrm{R} & 3.3 \mathrm{R}\end{array}$

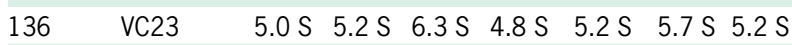
$137 \quad V C-26 \quad 4.1 \mathrm{~S} \quad 5.0 \mathrm{~S} \quad 6.3 \mathrm{~S} \quad 4.0 \mathrm{~S} \quad 4.9 \mathrm{~S} \quad 5.5 \mathrm{~S} \quad 4.8 \mathrm{~S}$

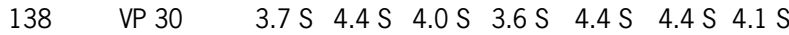

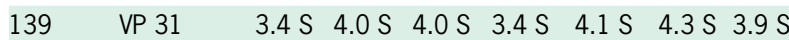

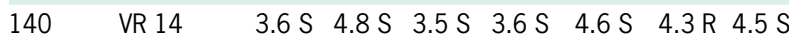
$141 \quad$ VR $15 \quad 3.7 \mathrm{~S} \quad 5.2 \mathrm{~S} \quad 6.3 \mathrm{~S} \quad 3.8 \mathrm{~S} \quad 5.0 \mathrm{~S} \quad 5.5 \mathrm{~S} \quad 4.8 \mathrm{~S}$ $\begin{array}{llllllllll}142 & V R & 16 & 3.7 \mathrm{~S} & 5.6 \mathrm{~S} & 6.0 \mathrm{~S} & 3.7 \mathrm{~S} & 5.3 \mathrm{~S} & 5.4 \mathrm{~S} & 4.8 \mathrm{~S}\end{array}$ $143 \quad$ VR $17 \quad 4.1 \mathrm{~S} \quad 5.7 \mathrm{~S} \quad 6.3 \mathrm{~S} \quad 4.1 \mathrm{~S} \quad 5.4 \mathrm{~S} \quad 5.6 \mathrm{~S} \quad 5.1 \mathrm{~S}$

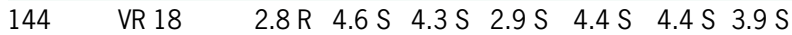
${ }^{*} \mathrm{R}=$ resistant; $\mathrm{S}=$ susceptible ${ }^{*}$ Lines from ten cycles of the recurrent selection program for resistance to angular leaf spot; ${ }^{1}$ Average scores; ${ }^{2}$ Predicted genotypic values (average BLUP).

Distribution of residuals of evaluations showed higher precision in the greenhouse (V2 and V3 stages) than in the field (Figure 3). Higher precision can be confirmed by accuracy estimates (Table 3).

Estimates of variance components associated to the effect of lines $\left(\hat{\sigma}_{g}^{2}=0.23\right)$ and genotype $\times$ environment interaction $\left(\hat{\sigma}_{f}^{2}=0.27\right.$ ) were significant (Table 3) considering the joint analysis involving all stages/environments. Significant interaction shows that reaction of the lines to mixture of $P$. griseola strains was not coincident in different environments of evaluation, on average. This can be confirmed by genetic correlation estimates that were low to medium magnitude (Table 3). Classification of predicted genotypic values of each environment changed the ranking of lines evidencing occurrence complex genotype $\times$ stages/ environment interactions and simple interaction (Figure 4).

Results of the analysis using the mixed model approach showed higher frequency of predicted genotypic values estimates from 3.1 to 4 in V2 stage; however, for V3 stage, in the field and joint analysis, estimates were 4.1 to 5 (Figure 5). Among 29 most resistant lines (lower BLUP average), $72 \%$ is from the recurrent selection program for ALS considering the joint analysis (Table 1). 

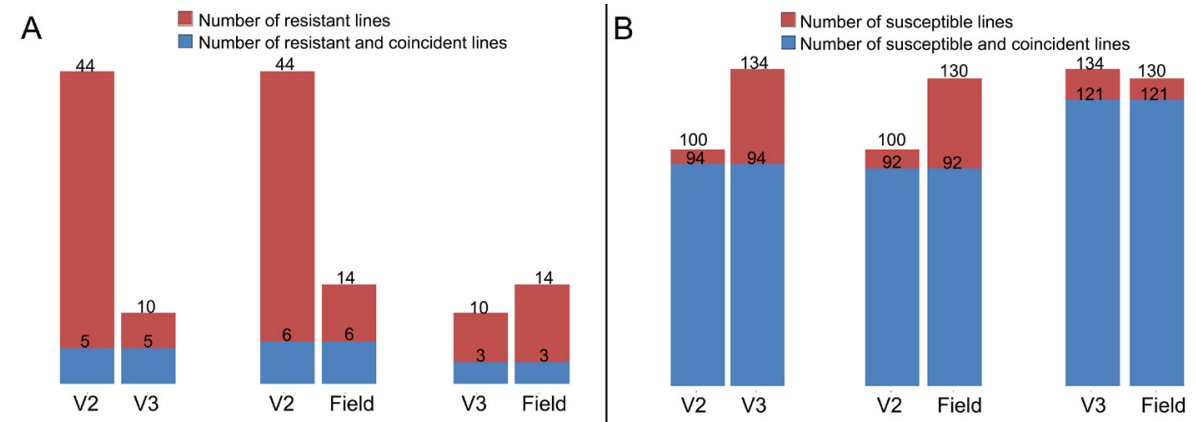

Figure 1 - A) Number of resistant lines and coincidence of resistant lines, considering the experiments pairwise; B) Number of susceptible lines and coincidence of susceptible lines, considering the experiments pairwise.

Table 2 - Number of resistant lines in each stage/environment of evaluation (diagonal); number of resistant and coincident lines in different stages/environments (above the diagonal) and name of these lines (below the diagonal).

\begin{tabular}{lccc}
\hline & & & \\
\hline V2 & V2 & V3 & Field \\
V3 & MAIX-4; CXII-15; MAIV-15.524; & 5 & 6 \\
& VC-28; PT-65 & 10 & 3 \\
Field & MAI-18.13; MAVIII-128; MAII- & PT-65; MAIV-8.102; MAIV- & 14 \\
& 10;MAVII-244; MAVIII-89; PT65 & 15.204 & \\
\hline
\end{tabular}

Table 3 - Estimates of variance components and genetic parameters for reaction of common bean lines to Pseudocercospora griseola in different stages/environments and in the joint analysis of stages/environments.

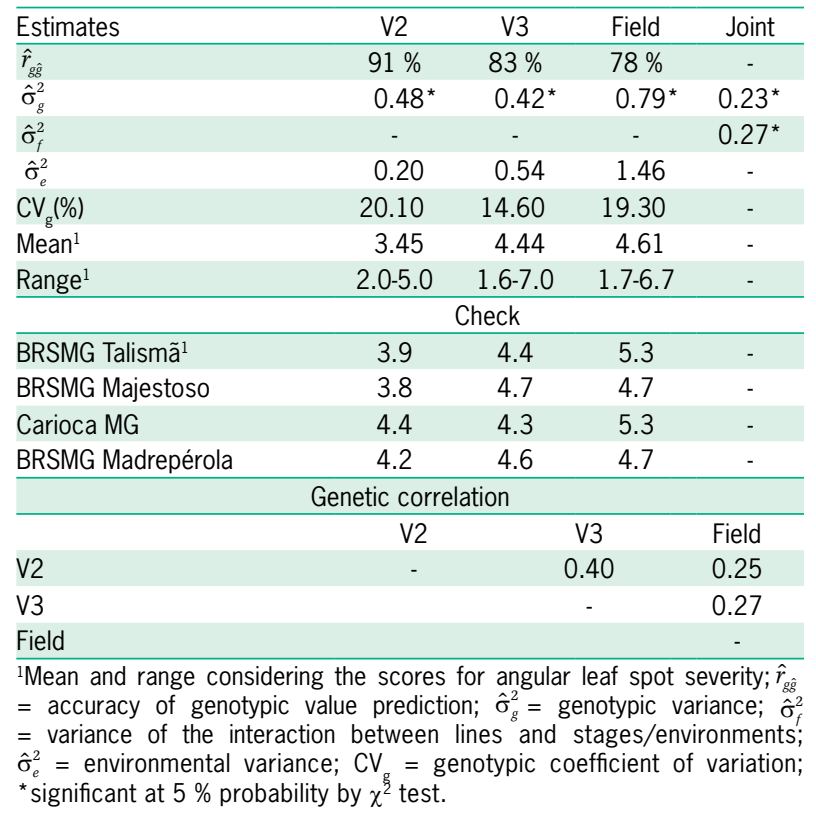

\section{Discussion}

Accurate phenotyping of ALS severity is important to identify new resistance sources to select progenies in breeding programs and to identify QTLs and molecular

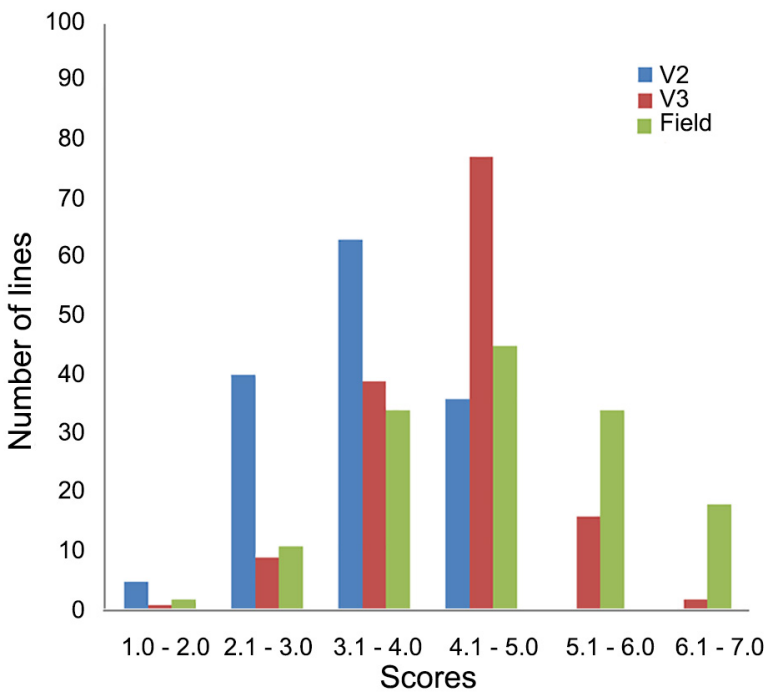

Figure 2 - Frequency of common bean lines according to score range (1 to 9) for angular leaf spot severity (ALS) assessed in different stages/environments.

markers for marker-assisted selection (Oblessuc et al., 2012; Rezende et al., 2014). There are several options in phenotyping, including assessment under controlled conditions or in the field and in different plant development stages. Thus, this study investigated whether different methodologies provide coinciding selection.

There were significant differences in ALS severity among genotypes in the analysis for stages/environments (Table 3). In this case, accuracy $\left(\hat{r}_{g \hat{g}}\right)$ of experiments and/or evaluations in the greenhouse /V2 and V3 stages) showed to be higher than in the field, as verified in distribution of residuals (Figure 3). Similar results were reported in evaluation of common bean progenies for resistance to white mold (Sclerotinia sclerotiorum (Lib.) de Bary) in the field and the greenhouse (Leite et al., 2016). This greater experimental precision is mainly due to temperature and humidity that were controlled in the greenhouse, providing favorable conditions for disease development. In addition, in the greenhouse, there was presence of mixture of two races only that were 
inoculated, ensuring greater uniformity of scores in different plots of the same treatment. In the field, despite the artificial inoculations, inoculum distribution was not as uniform as in the greenhouse and environmental conditions may not be favorable. Moreover, with natural pathogen occurrence, other races may appear with irregular distribution among plots of the same treatment, reducing accuracy.

We used common checks in all experiments. Cultivar Carioca MG showed susceptibility reaction in all evaluations, indicating favorable conditions for disease development (Table 3). Since the beginning of recurrent selection program of UFLA, cultivar Carioca MG has been used as susceptible control to ALS (Arantes et al.,

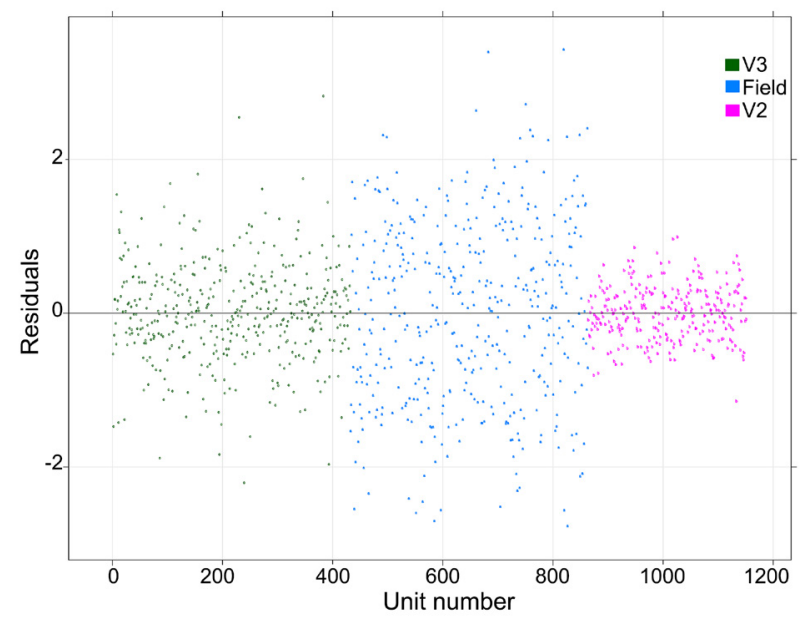

Figure 3 - Distribution of errors associated with scores for angular leaf spot (ALS) severity obtained in each stage/environment of evaluation.
2010; Rezende et al., 2014). Cultivars BRSMG Madrepérola and BRSMG Majestoso also were susceptible in all experiments, as reported in the literature (Pereira et al., 2015).

The mixed model was fit to carry out joint analyses because of heterogeneity of errors of different experiments, especially by lower errors estimates in experiments in the greenhouse. There was high concordance between the resistant lines using the classification obtained in both methodologies for all stages/environments of evaluation. The mixed model approach used in this work has been increasingly used in plant breeding, mainly due to its flexibility and robustness (Piepho et al., 2008).

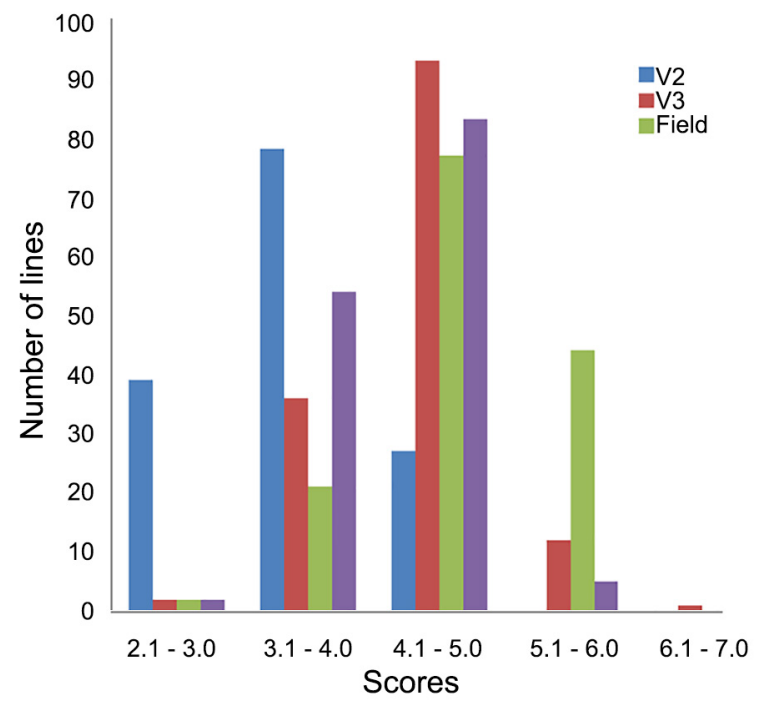

Figure $\mathbf{5}$ - Distribution of frequencies of predicted genotypic values for reaction of common bean lines to Pseudocercospora griseola, assessed in different environments/stages.

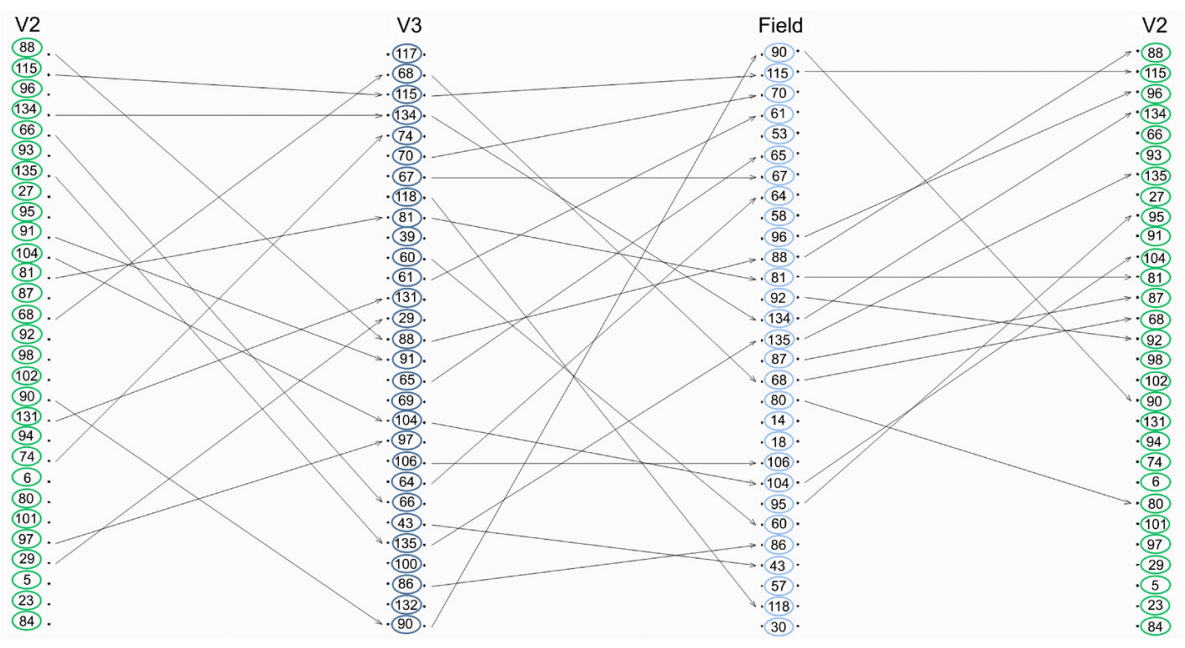

Figure 4 - Classification of predicted genotypic values of angular leaf spot (ALS) severity in each stage/environment. Numbers in the figure correspond to the lines described in Table 1. 
There was a pronounced effect of interaction lines $\times$ stages/environments of evaluation, since the associated variance component $\left(\hat{\sigma}_{f}^{2}=0.27\right)$ was greater than the estimated genetic variance between lines $\left(\hat{\sigma}_{g}^{2}=0.23\right)$. The decomposition of interaction component of the joint analysis showed that interaction was more pronounced when experiment in the field was involved. Therefore, the field experiment contributed more to interaction than the greenhouse did. Recent studies on common bean $P$. griseola pathosystem reported the predominance of quantitative resistance (Oblessuc et al., 2012; Pereira et al., 2015). Several genes control this resistance and expression of these genes may vary among environments. In this sense, individuals of different genotypes may differ in behavior depending on the environment, contributing to interaction (Falconer and MacKay, 1996). Thus, low genetic correlations between environments (Table 3 ) indicate that different genes express and/or have different levels of expression in the field and in the greenhouse, contributing to interaction (Falconer and MacKay, 1996; Ramalho et al., 2012).

Differential expression of genes in different environments may be explained by the occurrence of epigenetic events. Thus, different conditions of stress, abiotic and biotic, such as high temperatures and occurrence of other pathogens, may activate transposable elements or cause several epigenetic modifications. These modifications are stable in many cell divisions, however, they do not involve changes in DNA sequence of organism, contributing to expression of different genes under different stress conditions (Boyko and Kovalchuk, 2011; Rebollo et al., 2012; Bressan et al., 2014). DNA methylation in genomic regions associated to defense genes in tomato and rice have been reported in literature (Mason et al., 2008; Sha et al., 2005). These studies have shown a large number of methylations in DNA of infected adult plants than in seedlings. Thus, phenotyping in the field becomes very important because different environmental conditions activate different response mechanisms to stress.

Different conditions of biotic and abiotic stresses may explain the high magnitude of interaction lines $x$ stages/environments. For example, the possible existence of other races in the field, due to natural occurrence of pathogen. Moreover, environmental conditions of temperature and humidity are different in the field. Dry season is favorable for disease development and pathogen conidia are spread mainly through the wind (Liebenberg and Pretorius, 1997). Oblessuc et al. (2012) evaluated a population of recombinant lines (RILs) in the field (only the natural occurrence of the pathogen) and in the greenhouse and obtained similar results. Several QTLs were mapped in different linkage groups. However, among the identified QTLs, only one showed significant effect in both environments. Therefore, the genes expressed should be different, but few stable QTLs should be maintained. Low correlation between ALS severity scores in the field and greenhouse was mainly due to genotypes $x$ environments interaction. In this study, we carried out artificial inoculations in the field; thus, lower lines $\times$ environments interaction was expected, compared to the results found by Oblessuc et al. (2012). However, interaction was of higher magnitude.

There was higher ALS severity and a larger number of susceptible lines in the field than in the greenhouse. Evaluation in the field was conducted later (R7 stage), which may have contributed to the increase in disease severity. The suitable stage for assessing ALS severity in the field was studied by Rezende et al. (2014) that observed an increase in average scores of common bean lines along the evaluation periods. The most suitable stage for assessing the disease was $33 \mathrm{~d}$ after flowering, allowing greater discrimination among lines.

There was a high coincidence index for reaction of lines in V3 stage and in the field (88\%). However, only three lines were resistant in both environments. In both evaluation stages, plants present the so-called adult plant resistance that is mainly horizontal and has been described in several studies (Barcellos et al., 2000; Costa et al., 2006; Burdon et al., 2014). "Partial resistance" or horizontal resistance slows the process of infection, growth and pathogen reproduction in adult plants, but not in seedlings (Costa et al., 2006).

In this study, some lines, such as MAX-2 and VC 15 , presented resistance reaction in the greenhouse and were susceptible in the field. Similar results have been observed in evaluation of reaction of wheat progenies to Pyricularia grisea. (Cooke) Sacc. (Cruz et al., 2010). According to Bonman (1992), these differences may be attributed to different conditions of biotic and abiotic stresses and the presence of pathogen races in the field that were not inoculated in the greenhouse, as previously mentioned. Similar behavior has also been reported in the literature (Pereira et al., 2013; Pereira et al., 2015).

Susceptibility reaction in the greenhouse and resistance reaction in the field was also observed for five lines. Oblessuc et al. (2015) found susceptible responsive genes within the major PV10 QTL, which are different to the ones related to resistance. According to Barcellos et al. (1997), most genes that confer resistance to seedlings may be expressed throughout the plant cycle. However, other genes are only expressed in the adult stage (Burdon et al., 2014), as in wheat-Puccinia recondita f. sp. tritici (Eriks. \& E.Henn.) pathosystem (Barcellos et al., 1997).

Several genetic mapping studies have been conducted to identify QTLs related to ALS (Mahuku et al., 2009; Oblessuc et al., 2013; Keller et al., 2015; Perseguini et al., 2016). In these studies, the phenotyping of individuals is carried out only under controlled conditions (greenhouse). Therefore, it is necessary to verify for coincident behavior between QTLs identified in controlled conditions and in the field. In evaluations of lines belonging to common bean germplasm bank, it has already been identified various sources of resistance to ALS (Mahuku et al., 2003; Pádua et al., 2016). Similar to the identifica- 
tion procedure of QTLs, these evaluations are carried out only under controlled conditions, with inoculation of one or a few races pathogen. Thus, it is necessary to determine whether lines resistant under controlled conditions also have a reasonable level of resistance in the field.

Recurrent selection is a reliable method to accumulate favorable alleles, including those of small effects from different parents (Rezende et al., 2014; Nelson et al., 2018). This method has been successfully used for selection of progeny resistant to $P$. griseola (Amaro et al., 2007, Arantes et al., 2010). Most resistant lines in the field and greenhouse are from the recurrent selection program for ALS (V3 stage-50 \%; V2 stage - $59 \%$, field $-75 \%)$. One of steps of recurrent selection program is to assess progenies for selection of the most resistant. In the case of this program, evaluation of disease to select progenies is carried out in field, in dry season, when conditions are favorable for disease development (Amaro et al., 2007). Therefore, lines from the recurrent selection program performed better in the field, since they were selected for several cycles in this environment. The joint analysis using the mixed model approach showed that 72 $\%$ of resistant lines derive from the recurrent selection program to ALS resistance. Lines from this program, in general, have presented good resistance level to $P$. griseola, regardless of the environment (Arantes et al., 2010; Rezende et al., 2014; Pereira et al., 2015).

Evaluation in V2 stage selected a larger number of lines and may be considered a moderate selection. In germplasm banks, there are many accesses to be characterized. Thus, screening in V2 stage could be used because, at this stage of evaluation, a shorter and smaller amount of inoculum is used in inoculation (Librelon et al., 2015). Therefore, a pre-selection is made, discarding the most susceptible genotypes in stage V2 and after, a more careful evaluation is carried out using evaluation in V3 stage and, if possible, field evaluation. Furthermore, in a recurrent selection program, evaluation of progenies in V2 stage allows more than one cycle per year, increasing genetic gain. In this case, progenies are sown in trays, inoculated and resistant plants are transplanted to pots to carry out the recombination step. Therefore, it is possible to carry out selection of progenies in that greenhouse and in the field in dry season (Amaro et al., 2007; Arantes et al., 2010; Librelon et al., 2015). Another alternative is to pre-select progenies in V2 stage to assess resistant plants in the field and then perform recombination.

Our findings show that evaluation in V3 stage and in the field were more efficient phenotyping methods for ALS severity. However, evaluation in V3 stage is unfeasible to assess routinely many genotypes in breeding programs due to the limited physical space and amount of pathogen inoculum. Inoculation in V2 stage allows evaluating a large number of genotypes. In this study, there was high coincidence among susceptible lines selected in all methods (Figure 1B). Therefore, evaluation in V2 stage may be used, especially in early stages of breeding programs, to carry out a first screening.

\section{Acknowledgements}

The authors thank the Coordenação de Aperfeiçoamento de Pessoal de Nível Superior (CAPES), Fundação de Amparo à Pesquisa do Estado de Minas Gerais (FAPEMIG), and Conselho Nacional de Desenvolvimento Cientifico e Tecnológico (CNPq), for granting a scholarship and for funding the project.

\section{Authors' Contributions}

Conceptualization: Pereira, R.; Souza, E.A.; Abreu, A.F.B. Data acquisition: Pereira, R.; Souza, E.A.; Abreu, A.F.B. Data analysis: Pereira, R.; Nalin, R.S. Design of methodology: Pereira, R.; Souza, E.A.; Abreu, A.F.B.; Nalin, R.S. Writing and editing: Pereira, R.; Souza, E.A.; Abreu, A.F.B.; Nalin, R.S.

\section{References}

Amaro, G.B.; Abreu, A.F.B.; Ramalho, M.A.P. 2007. Phenotypic recurrent selection in the common bean (Phaseolus vulgaris L) with carioca-type grains for resistance to the fungi Phaeoisariopsis griseola. Genetics and Molecular Biology 30: 584-588.

Arantes, L.O.; Abreu, A.F.B.; Ramalho, M.A.P. 2010. Eight cycles of recurrent selection for resistance to angular leaf spot in common bean. Crop Breeding and Applied Biotechnology 10: 232-237.

Barcellos, A.L.; Moraes-Fernandes, M.I.B.; Roelfs, A.P. 1997. Wheat leaf rust (Puccinia recondita): resistance durability. Summa Phytopathologica 23: 101-111 (in Portuguese, with abstract in English).

Barcellos, A.L.; Roelfs, A.P.; Moraes-Fernandes, M.I.B. 2000. Inheritance of adult plant leaf rust resistance in the Brazilian wheat cultivar toropi. Plant Disease 84: 90-93.

Boyko, A.; Kovalchuk, I. 2011. Genome instability and epigenetic modification: heritable responses to environmental stress? Current Opinion in Plant Biology 14: 260-266.

Bonman, J.M. 1992. Durable resistance to rice blast diseaseenvironmental influences. Euphytica 63: 115-123.

Borel, J.C.; Ramalho, M.A.P.; Abreu, A.F.B.; Maia, L.G.S. 2011. Genetic control of the angular leaf spot reaction in common bean leaves and pods. Scientia Agricola 68: 661-664.

Bressan, R.A.; Jian-Kang, Z.; Van Oosten, M.J.; Maggio, A.; Bohnert, H.J.; Chinnusamy, V. 2014. Epigenetics connects the genome to its environment. Plant Breeding Reviews 38: 69-142.

Burdon, J.J.; Barrett, L.G.; Rebetzke, G.; Thrall, P.H. 2014. Guiding deployment of resistance in cereals using evolutionary principles. Evolutionary Applications 7: 609-624.

Costa, I.F.D.D.; Balardin, R.S.; Medeiro, L.A.; Bayer, T.M. 2006. Resistance of six soybean cultivars to Colletotrichum truncatum (Schwein) in two phenologic stages. Ciência Rural 36: 1684-1688 (in Portuguese, with abstract in English).

Chiorato, A.F.; Carbonell, S.A.M.; Dias, L.A.D.S.; Rezende, M.D.V.D. 2008. Prediction of genotypic values and estimation of genetic parameters in common bean. Brazilian Archives of Biology and Technology 51: 465-472. 
Cruz, M.A.F.; Prestes, A.M.; Maciel, J.L.N.; Scheeren, P.L. 2010. Partial resistance to blast on common and synthetic wheat genotypes in seedling and in adult plant growth stages. Tropical Plant Pathology 35: 024-031 (in Portuguese, with abstract in English).

Falconer, D.S.; MacKay, T.F.C. 1996. Introduction to Quantitative Genetics. Longman, London, UK.

Jara, C.; Pastor, M.A.; Mahuku, G.; Mosquera, G. 2015. Virulence diversity of Pseudocercospora griseola and its implication for breeding common bean for resistance to angular leaf spot. Annual Report Bean Improvement Cooperative 59: 38-116.

Keller, B.; Manzanares, C.; Jara, C.; Lobaton, J.D.; Studer, B.; Raatz, B. 2015. Fine-mapping of a major QTL controlling angular leaf spot resistance in common bean (Phaseolus vulgaris L.). Theoretical and Applied Genetics 128: 813-826.

Leite, M.E.; Dias, J.A.; Souza, D.A.D.; Alves, F.C.; Pinheiro, L.R.; Santos, J.B.D. 2016. Increasing the resistance of common bean to white mold through recurrent selection. Scientia Agricola 73: 71-78.

Librelon, S.S.; Souza, E.A.; Pereira, R.; Pozza, E.A.; Abreu, A.F.B. 2015. Diagrammatic scale to evaluate angular leaf spot severity in primary leaves of common bean. Australasian Plant Pathology 44: 385-395.

Liebenberg, M.M.; Pretorius, Z.A. 1997. A review of angular leaf spot of common bean (Phaseolus vulgaris L.). African Plant Protection 3: 81-106.

Mahuku, G.S.; Iglesias, A.M.; Jara, C. 2009. Genetics of angular leaf spot resistance in the Andean common bean accession G5686 and identification of markers linked to the resistance genes. Euphytica 167: 381-396.

Mahuku, G.S.; Jara, C.; Cajiao, C.; Beebe, S. 2003. Sources of resistance to angular leaf spot (Phaeoisariopsis griseola) in common bean core collection, wild Phaseolus vulgaris and secondary gene pool. Euphytica 130: 303-313.

Mason, G.; Noris, E.; Lanteri, S.; Acquadro, A.; Accotto, G.P.; Portis, E. 2008. Potentiality of methylation-sensitive amplification polymorphism (MSAP) in identifying genes involved in tomato response to tomato yellow leaf curl sardinia virus. Plant Molecular Biology Reporter 26: 156-173.

Nelson, R.; Wiesner-Hanks, T.; Wisser, R.; Balint-Kurti P. 2018. Navigating complexity to breed disease-resistant crops. Nature Reviews Genetics 19: 21-33.

Oblessuc, P.R.; Perseguini, J.M.K.C.; Baroni, R.M.; Chiorato, A.F.; Carbonell, S.A.M.; Mondego, J.M.C.; Benchimol-Reis, L.L. 2013. Increasing the density of markers around a major QTL controlling resistance to angular leaf spot in common bean. Theoretical and Applied Genetics 126: 2451-2465.

Oblessuc, P.R.; Baroni, R.M.; Garcia, A.A.F.; Chioratto, A.F.; Carbonell, S.A.M.; Camargo, L.E.A.; Benchimol, L.L. 2012. Mapping of angular leaf spot resistance QTL in common bean (Phaseolus vulgaris L.) under different environments. BMC Genetics 13: 1-9.

Oblessuc, P.R.; Matiolli, C.C.; Chiorato, A.F.; Camargo, L.E.A.; Reis, L.L.B.; Melotto, M. 2015. Common bean reaction to angular leaf spot comprises transcriptional modulation of genes in the ALS10.1 QTL. Frontiers in Plant Science 6: 1-11.
Pádua, P.F.; Barcelos, Q.L.; Souza, E.A.; Abreu, A.F.B. 2016. Identification of common bean resistant sources to angular leaf spot disease in a Brazilian germplasm collection. Annual Report of the Bean Improvement Cooperative 59: 115-116.

Pereira, R.; Souza, E.A.; Barcelos, Q.L.; Abreu, A.F.B.; Librelon, S.S. 2015. Aggressiveness of Pseudocercospora griseola strains in common bean genotypes and implications for genetic improvement. Genetics and Molecular Research 14: 50445053.

Pereira, R.; Souza, E.A.; Barcelos, Q.L.; Abreu, A.F.B. 2013. Evaluation of resistance in common bean genotypes to the causal agent of angular leaf spot. Annual Report of the Bean Improvement Cooperative 56: 33-34.

Pereira, R.; Abreu, M.J.; Souza, E.A. 2011. Alternative method to assess the reaction of common bean lines to Pseudocercospora griseola. Annual Report of the Bean Improvement Cooperative 54: 104-105.

Perseguini, J.M.K.C.; Oblessuc, P.R.; Rosa, J.R.B.F.; Gomes, K.A.; Chiorato, A.F.; Carbonell, S.A.M.; Benchimol-Reis, L.L. 2016. Genome-wide association studies of anthracnose and angular leaf spot resistance in common bean (Phaseolus vulgaris L.). PloS One 11: e0150506.

Piepho, H.P.; Möhring, J.; Melchinger, A.E.; Büchse, A. 2008. BLUP for phenotypic selection in plant breeding and variety testing. Euphytica 161: 209-228.

Ramalho, M.A.P.; Abreu, A.F.B.; Santos, J.B.; Nunes. J.A.R. 2012. Quantitative Genetics in Plant Breeding = Aplicações da Genética Quantitativa no Melhoramento de Plantas Autógamas. UFLA, Lavras, MG, Brazil (in Portuguese).

Rezende, B.A.; Abreu, A.F.B.; Ramalho, M.A.P.; Souza, E.A. 2014. Severity evaluation methods in common bean recurrent selection programme for resistance to angular leaf spot. Journal of Phytopathology 162: 643-649.

Rodriguez, I.J.; Vargas, A.G.; Rosas, J.C.; Beaver, S.B.; Porch, T.G. 2014. Resistance of common bean breeding lines to Phaeoisariopsis griseola isolates from Honduras. Annual Report of the Bean Improvement Cooperative 57: 215-216.

Rebollo, R.; Romanish, M.T.; Mager, D.L. 2012. Transposable elements: an abundant and natural source of regulatory sequences for host genes. Annual Review Genetics 46: 21-42.

Sha, A.H.; Lin, X.H.; Huang, J.B.; Zhang, D.P. 2005. Analysis of DNA methylation related to rice adult plant resistance to bacterial blight based on methylation-sensitive AFLP (MSAP) analysis. Molecular Genetics and Genomics 273: 484-490.

Silva, K.J.D.; Freire, C.N.S.; Souza, E.A. 2008. Pathogenic variability of isolates of Pseudocercospora griseola, the cause of common bean angular leaf spot, and its implications for resistance breeding. Journal of Phytopathology 156: 602-606.

Steel, R.G.D.; Torrie, J.H.; Dickey, D.A. 1997. Principles and procedures of statistics: a biometrical approach. McGraw-Hill, New York, NY, USA.

Van Schoonhoven, A.; Pastor-Corrales, M.A. 1987. Standard System for the Evaluation of Bean Germplasm. CIAT, Cali, Colombia. 